\title{
Effect of nitrogen rate and application on growth and yield of okra (Abelmoschus esculentus L.) under rain- fed conditions at Blue Nile State, Sudan
}

\author{
Adlan M. A. Adlan ${ }^{1 \star}$, M. A. Adlan ${ }^{2}$, Eisa Y. A. ${ }^{1}$ and Amin El Sir A. I. ${ }^{1}$ \\ ${ }^{1}$ Damazine Research Station, Sudan. \\ ${ }^{2}$ Gezira Research Station, Sudan.
}

Accepted 6 November, 2017

\begin{abstract}
Experiments were carried out at Damazin Research Station farm during kharif season 2014 and 2016, to evaluate the effects of nitrogen rate and split doses on growth and yield of okra under rain-fed condition. Treatments include factorial combinations of three nitrogen rates viz 43, 86 and $129 \mathrm{~kg} \mathrm{~N} \mathrm{ha}^{-1}$ and four application rates viz at 1,2, 3 and 4 splits doses and an un-treated control. Results show that nitrogen doses effected plant height in two seasons, while split doses and their interaction were affected only in season two. Results showed that nitrogen doses, split doses and there interaction were evident in fresh and dry yield tons ha ${ }^{-1}$ over two seasons.
\end{abstract}

Keywords: Plant height, yield, nitrogen, phosphorus, harvest.

${ }^{*}$ Corresponding author. E-mail: adlan.m.a.2015@gmail.com.

\section{INTRODUCTION}

Okra (Abelmoschus esculentus L. (Moench) is an economically important vegetable crop grown in tropical and sub-tropical parts of the world. World okra production in 2010 was 6.9 million tons, while in the Sudan okra production was estimated to amount to 2.6 million tons (FAOSTAT, 2010). The crop is suitable for cultivation as a garden crop as well as on large commercial farms (Tripathi et al., 2011). The nutritional composition of okra includes calcium, protein, fat and carbohydrates, iron, magnesium and phosphorus (Omotoso and Shittu, 2007). Akinfasoye and Nwanguma (2005) noted that the oil in the seed could be as high as inpoultry eggs and soybean.

It is consumed by almost all the Sudanese people either as green immature pods (fried, cooked or in soup and stews) or sun dried and ground into a powdery form locally known as wieka" which is used as an ingredient in the preparation of a favorable Sudanese molah (Osman, 2005). Among improvement possibilities, the nutritional requirements play a major role. Nitrogen, Phosphorus and Potassium are major essential elements required for physiological mechanisms of plant growth (Rao and
Subramanian, 1994). Nitrogen and phosphorus are usually the most limiting nutrients in many soils in Africa and are often simultaneously deficient. Most soils in SubSahara African are used for subsistence farming and are of low and declining fertility (Buresh et al., 1997). Continuous cropping with low or no fertilizer inputs, nutrient losses through harvest, soil erosion and leaching has led to decline in soil fertility (Cooper et al., 1996). No research has been done on the performance of okra to nitrogen fertilizer under rain-fed conditions in the Blue Nile State. The present study was undertaken to evaluate the performance of okra to different nitrogen levels and split rates under rain-fed conditions in the Blue Nile State.

\section{MATERIALS AND METHODS}

Experiments were carried out at the farm of the Damazin Research Station during kharif season 2014 and 2016, in order to evaluate the effects of nitrogen rate and split doses on growth and yield of okra under rain-fed condition. Treatments included factorial combinations of three nitrogen rates viz 43, 86 and $129 \mathrm{~kg} \mathrm{~N} \mathrm{ha}^{-1}$ 
and four splits doses viz at 1, 2, 3 and 4 and un-treated control (Table 1). The land was first disc harrowed and then divided into raised beds $80 \mathrm{~cm}$ wide the experimental units area were four ridges of $4 \mathrm{~m}$ long $\left(12.8 \mathrm{~m}^{2}\right)$. Sowing dates were on $19^{\text {th }}$ and $18^{\text {th }}$ July over two seasons respectively, while the $13^{\text {th }}$ treatments were arranged in a randomized complete block design (RCBD) with 3 replications. Four or three seeds were planted at a spacing of 20 $\mathrm{cm}$ and then thinned to two plants per hill. Hand weeding and pest control were used when necessary. The data collected included growth, yield parameters and pod characters of okra. Five plants were taken randomly to measured growth parameters at the $50 \%$ flowering stage including stem length and diameter, number of branches and leaves plant ${ }^{-1}$. Yield parameters were measured at the time of harvest and they included number, fresh and dry yield. The data were subjected to ANOVA using the GenStat computer package (Buysse et al., 2004).

Table 1. Combined analysis and interaction effects of urea doses and split applications on okra plant height (cm) over two seasons under rain-fed condition at Damazin Research Station.

\begin{tabular}{|c|c|c|c|c|c|c|c|c|c|c|c|c|}
\hline \multirow{3}{*}{$\mathbf{N}$ levels (kg ha ${ }^{-1}$ ) } & \multicolumn{6}{|c|}{ Season two } & \multicolumn{6}{|c|}{ Season one } \\
\hline & \multicolumn{6}{|c|}{ Splits doses } & \multicolumn{6}{|c|}{ Splits doses } \\
\hline & 0 & 1 & 2 & 3 & 4 & Mean & 0 & 1 & 2 & 3 & 4 & Mean \\
\hline 0 & 111.0 & & & & & 111.0 & 55.3 & & & & & 55.3 \\
\hline 43 & & 131.2 & 133.8 & 135.3 & 123.7 & 131.0 & & 61. & 66.0 & 57.0 & 56.0 & 60.1 \\
\hline 86 & & 134.3 & 146.5 & 139.8 & 142.8 & 140.8 & & 55.7 & 63.3 & 69.3 & 61.3 & 62.4 \\
\hline 129 & & 133.1 & 140.1 & 145.6 & 135.5 & 138.6 & & 66. & 70.7 & 77.5 & 58.3 & 68.2 \\
\hline Mean & 111.0 & 132.8 & 140.1 & 140.2 & 134.0 & & 55.3 & 61. & 66.7 & 67.9 & 58.5 & \\
\hline Statistics & \multicolumn{2}{|c|}{ Urea doses } & \multicolumn{3}{|c|}{ Splits doses } & teraction & \multicolumn{2}{|c|}{ Urea doses } & \multicolumn{2}{|c|}{ Splits doses } & \multicolumn{2}{|c|}{ Interaction } \\
\hline Sig. & \multicolumn{2}{|c|}{ * } & \multicolumn{2}{|c|}{ NS } & & NS & \multicolumn{2}{|c|}{$* * *$} & \multicolumn{2}{|c|}{$* * *$} & \multicolumn{2}{|c|}{$\star \star \star *$} \\
\hline SE \pm & \multirow{2}{*}{\multicolumn{2}{|c|}{3.1}} & \multirow{2}{*}{\multicolumn{2}{|c|}{$5.5^{-}$}} & & - & \multirow{2}{*}{\multicolumn{4}{|c|}{$\begin{array}{r}1.6 \\
5.5\end{array}$}} & \multirow{2}{*}{\multicolumn{2}{|c|}{2.9}} \\
\hline CV\% & & & & & & & & & & & & \\
\hline
\end{tabular}

\section{RESULTS}

Table 1 shows that nitrogen rates had an effect on plant height in both seasons, while split rates and its interactions were effects only in season two. The greatest plant height was obtained by $86 \mathrm{~kg} \mathrm{ha}^{-1}$ and $129 \mathrm{~kg} \mathrm{ha}^{-1}$ nitrogen in both seasons. The greatest plant height in season two was obtained by split application of nitrogen in two and three equal rates. An interaction of $129 \mathrm{~kg} \mathrm{ha}^{-1}$ nitrogen rate with a split application in two and three equal doses.

Table 2 shows that nitrogen doses, split doses and its interactions did not affect the number of branches plant ${ }^{-1}$ except for nitrogen rates in season two. The highest number of branches plant ${ }^{-1}$ in season two was obtained by $86 \mathrm{~kg} \mathrm{ha}^{-1}$ and $129 \mathrm{~kg} \mathrm{ha}^{-1}$ treatments, respectively.

Table 3 shows that nitrogen, split rates and its interactions showed effects on the number of leaves plant $^{-1}$ except for split rates in season one. The highest number of leaves plant ${ }^{-1}$ in season one was obtained by a nitrogen treatment of $86 \mathrm{~kg} \mathrm{ha}^{-1}$ and interactions with split application of three rates. The greatest number of leaves plant ${ }^{-1}$ in season two was obtained by a nitrogen rate of $129 \mathrm{~kg} \mathrm{ha}{ }^{-1}$, split application of nitrogen in three equal rates.

Table 4 shows that nitrogen rates, split application and its interactions significantly increased the number of pods plant $^{-1}$ except for no interaction in season two. The greatest number of pods plant ${ }^{-1}$ in two seasons was obtained by the $129 \mathrm{~kg} \mathrm{ha}^{-1}$ nitrogen rate, with a split of application in three equal rates.

Table 5 shows that nitrogen rates, split application and its interactions significantly increased the fresh yield tons $\mathrm{ha}^{-1}$ in two seasons. The heist fresh yield tons ha ${ }^{-1}$ in two seasons was obtained by $86 \mathrm{~kg} \mathrm{ha}^{-1}$ and $129 \mathrm{~kg} \mathrm{ha}^{-1}$ nitrogen rate, with a split of application in three equal rates.

Table 6 shows that nitrogen rates, split application and its interactions significantly increased the dry yield tons $\mathrm{ha}^{-1}$ in two seasons. The heist dry yield tons ha $\mathrm{ha}^{-1}$ in two seasons was obtained by $86 \mathrm{~kg} \mathrm{ha}^{-1}$ and $129 \mathrm{~kg} \mathrm{ha}^{-1}$ nitrogen rate, with a split of application in three equal rates.

\section{DISCUSSION}

The greatest plant height was obtained by nitrogen application of $86 \mathrm{~kg} \mathrm{ha}^{-1}$ and $129 \mathrm{~kg} \mathrm{ha}^{-1}$ in both seasons. This is a result of the higher nitrogen content which induced higher plant height and more leaves. This agrees with the findings of Kolawole et al. (2008) who reported that increasing the rate of a NPK fertilizer, led to an increase in growth parameters of okra.

Fresh and dry fruit yield in tons $\mathrm{ha}^{-1}$ were lowest without the application of $\mathrm{N}$ fertilizer in both two seasons. These results are in accordance with the findings of Kolawole et al. (2008) that increasing the rate of NPK fertilizer would cause an increase in the performance of okra. 
Table 2. Combined analysis and interaction effects of urea doses and split applications on okra number of branches plant ${ }^{-1}$ over two seasons under rain-fed condition at Damazin Research Station.

\begin{tabular}{|c|c|c|c|c|c|c|c|c|c|c|c|c|}
\hline \multirow{3}{*}{ N levels (kg ha $\left.{ }^{-1}\right)$} & \multicolumn{6}{|c|}{ Season two } & \multicolumn{6}{|c|}{ Season one } \\
\hline & \multicolumn{6}{|c|}{ Splits doses } & \multicolumn{6}{|c|}{ Splits doses } \\
\hline & 0 & 1 & 2 & 3 & 4 & Mean & 0 & 1 & 2 & 3 & 4 & Mean \\
\hline 0 & 4.8 & & & & & 4.8 & 7.3 & & & & & 7.3 \\
\hline 43 & & 4.6 & 4.4 & 4.8 & 5.3 & 4.8 & & 7.5 & 8.2 & 7.6 & 6.4 & 7.4 \\
\hline 86 & & 4.7 & 4.8 & 4.7 & 4.8 & 4.7 & & 8.1 & 7.7 & 8.9 & 8.6 & 8.3 \\
\hline 129 & & 4.6 & 4.9 & 5.6 & 5.4 & 5.1 & & 8.9 & 7.9 & 9.0 & 9.1 & 8.7 \\
\hline Mean & 4.8 & 4.7 & 4.7 & 4.9 & 5.2 & & 7.3 & 8.1 & 8.0 & 8.5 & 8.0 & \\
\hline Statistics & \multicolumn{2}{|c|}{ Urea doses } & \multicolumn{2}{|c|}{ Splits doses } & \multicolumn{2}{|c|}{ Interaction } & \multicolumn{2}{|c|}{ Urea doses } & \multicolumn{2}{|c|}{ Splits doses } & \multicolumn{2}{|c|}{ Interaction } \\
\hline Sig. & \multicolumn{2}{|c|}{ NS } & \multicolumn{2}{|c|}{ NS } & \multicolumn{2}{|c|}{ NS } & * & & \multicolumn{2}{|c|}{ NS } & \multicolumn{2}{|c|}{ NS } \\
\hline $\mathrm{SE} \pm$ & - & & - & & - & & 0. & & 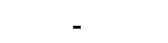 & & - & \\
\hline CV\% & \multicolumn{6}{|c|}{10.6} & \multicolumn{6}{|c|}{10.2} \\
\hline
\end{tabular}

Table 3. Combined analysis and interaction effects of urea doses and split applications on okra number of leaves plant ${ }^{-1}$ over two seasons under rain-fed condition at Damazin Research Station.

\begin{tabular}{|c|c|c|c|c|c|c|c|c|c|c|c|c|}
\hline \multirow{3}{*}{$\mathrm{N}$ levels (kg ha ${ }^{-1}$ ) } & \multicolumn{6}{|c|}{ Season two } & \multicolumn{6}{|c|}{ Season one } \\
\hline & \multicolumn{6}{|c|}{ Splits doses } & \multicolumn{6}{|c|}{ Splits doses } \\
\hline & 0 & 1 & 2 & 3 & 4 & Mean & 0 & 1 & 2 & 3 & 4 & Mean \\
\hline 0 & 19.1 & & & & & 19.1 & 12.9 & & & & & 12.9 \\
\hline 43 & & 22.0 & 20.2 & 22.8 & 21.3 & 21.6 & & 14.6 & 15.8 & 14.1 & 14.1 & 14.7 \\
\hline 86 & & 19.3 & 19.6 & 22.2 & 19.8 & 20.2 & & 13.7 & 15.4 & 17.1 & 15.6 & 15.5 \\
\hline 129 & & 18.4 & 20.0 & 16.2 & 20.8 & 18.9 & & 16.2 & 17.1 & 18.8 & 15.6 & 17.0 \\
\hline Mean & 19.1 & 19.9 & 19.9 & 20.4 & 20.6 & & 12.9 & 14.8 & 16.1 & 16.7 & 15.1 & \\
\hline Statistics & \multicolumn{2}{|c|}{ Urea doses } & \multicolumn{2}{|c|}{ Splits doses } & \multicolumn{2}{|c|}{ Interaction } & \multicolumn{2}{|c|}{ Urea doses } & \multicolumn{2}{|c|}{ Splits doses } & \multicolumn{2}{|c|}{ Interaction } \\
\hline Sig. & \multicolumn{2}{|c|}{ * } & \multicolumn{2}{|c|}{ NS } & \multicolumn{2}{|c|}{ * } & \multicolumn{2}{|c|}{$* * *$} & \multicolumn{2}{|c|}{$* * *$} & \multicolumn{2}{|c|}{ * } \\
\hline $\mathrm{SE} \pm$ & \multirow{2}{*}{\multicolumn{2}{|c|}{0.7}} & \multirow{2}{*}{\multicolumn{2}{|c|}{$\begin{array}{l}- \\
-\end{array}$}} & \multirow{2}{*}{\multicolumn{2}{|c|}{1.4}} & \multirow{2}{*}{\multicolumn{2}{|c|}{0.3}} & \multirow{2}{*}{\multicolumn{2}{|c|}{$\begin{array}{c}0.4 \\
5.1\end{array}$}} & \multirow{2}{*}{\multicolumn{2}{|c|}{0.7}} \\
\hline CV\% & & & & & & & & & & & & \\
\hline
\end{tabular}

Table 4. Combined analysis and interaction effects of urea doses and split applications on okra number of pods plant ${ }^{-1}$ over two seasons under rain-fed condition at Damazin Research Station.

\begin{tabular}{|c|c|c|c|c|c|c|c|c|c|c|c|c|}
\hline \multirow{3}{*}{$\mathbf{N}$ levels (kg ha ${ }^{-1}$ ) } & \multicolumn{6}{|c|}{ Season two } & \multicolumn{6}{|c|}{ Season one } \\
\hline & \multicolumn{6}{|c|}{ Splits doses } & \multirow[b]{2}{*}{0} & \multirow[b]{2}{*}{1} & \multirow[b]{2}{*}{2} & \multirow[b]{2}{*}{3} & \multirow[b]{2}{*}{4} & \multirow[b]{2}{*}{ Mean } \\
\hline & 0 & 1 & 2 & 3 & 4 & Mean & & & & & & \\
\hline 0 & 9.9 & \multirow[b]{2}{*}{10.6} & & & & 9.9 & 3.3 & \multirow[b]{2}{*}{5.2} & & & & 3.3 \\
\hline 43 & & & 11.4 & 11.8 & 11.1 & 11.2 & & & 5.1 & 6.1 & 5.3 & 5.4 \\
\hline 86 & & 11.9 & 13.1 & 12.4 & 10.9 & 12.1 & & 6.2 & 8.9 & 9.6 & 10.8 & 8.9 \\
\hline 129 & & 12.8 & 12.2 & 12.4 & 14.3 & 12.9 & & 10.6 & 10.9 & 10.6 & 7.9 & 10.0 \\
\hline Mean & 9.9 & 11.8 & 12.3 & 12.2 & 12.1 & & 3.3 & 7.3 & 8.3 & 8.8 & 8.0 & \\
\hline Statistics & \multicolumn{2}{|c|}{ Urea doses } & \multicolumn{2}{|c|}{ Splits doses } & \multicolumn{2}{|c|}{ Interaction } & \multirow{2}{*}{\multicolumn{2}{|c|}{$\underset{* \star *}{\text { Urea doses }}$}} & Splits doses & & \multicolumn{2}{|c|}{ Interaction } \\
\hline Sig. & \multicolumn{2}{|c|}{$* * *$} & \multicolumn{2}{|c|}{ * } & \multicolumn{2}{|c|}{$* * *$} & & & \multicolumn{2}{|l|}{ * } & \multicolumn{2}{|c|}{ NS } \\
\hline $\mathrm{SE} \pm$ & \multirow{2}{*}{\multicolumn{2}{|c|}{0.3}} & \multirow{2}{*}{\multicolumn{2}{|c|}{8.7}} & \multicolumn{2}{|c|}{0.7} & 0.2 & \multirow{2}{*}{\multicolumn{3}{|c|}{9.6}} & \multirow{2}{*}{\multicolumn{2}{|c|}{-}} \\
\hline CV\% & & & & & & & & & & & & \\
\hline
\end{tabular}


Table 5. Combined analysis and interaction effects of urea doses and split applications on okra fresh yield tons ha ${ }^{-1}$ over two seasons under rain-fed condition at Damazin Research Station.

\begin{tabular}{|c|c|c|c|c|c|c|c|c|c|c|c|c|}
\hline \multirow{3}{*}{$\mathrm{N}$ levels (kg ha ${ }^{-1}$ ) } & \multicolumn{6}{|c|}{ Season two } & \multicolumn{6}{|c|}{ Season one } \\
\hline & \multicolumn{6}{|c|}{ Splits doses } & \multicolumn{6}{|c|}{ Splits doses } \\
\hline & 0 & 1 & 2 & 3 & 4 & Mean & 0 & 1 & 2 & 3 & 4 & Mean \\
\hline 0 & 9.9 & & & & & 9.9 & 6.1 & & & & & 6.1 \\
\hline 43 & & 11.8 & 10.4 & 12.9 & 11.0 & 11.5 & & 9.4 & 9.2 & 11.1 & 9.7 & 9.8 \\
\hline 86 & & 13.6 & 14.3 & 14.9 & 9.6 & 13.1 & & 11.2 & 13.1 & 15.4 & 14.9 & 13.6 \\
\hline 129 & & 12.6 & 14.1 & 14.9 & 13.5 & 13.8 & & 15.5 & 15.9 & 15.4 & 11.5 & 14.6 \\
\hline Mean & 9.9 & 12.7 & 12.9 & 14.2 & 11.3 & & 6.1 & 12.0 & 12.7 & 14.0 & 12.0 & 12.2 \\
\hline Statistics & \multirow{2}{*}{\multicolumn{2}{|c|}{ Urea doses $_{* \star \star}$}} & \multicolumn{2}{|c|}{ Splits doses } & \multicolumn{2}{|c|}{ Interaction } & \multicolumn{2}{|c|}{ Urea doses } & \multicolumn{2}{|c|}{ Splits doses } & \multicolumn{2}{|c|}{ Interaction } \\
\hline Sig. & & & \multicolumn{2}{|c|}{$* * *$} & \multicolumn{2}{|c|}{$* * *$} & \multirow{2}{*}{\multicolumn{2}{|c|}{$\begin{array}{l}* * * \\
0.4\end{array}$}} & \multicolumn{2}{|c|}{ * } & \multicolumn{2}{|c|}{$* * *$} \\
\hline SE \pm & \multicolumn{2}{|c|}{0.4} & 0.9 & & 1.6 & & & & 0.5 & & & \\
\hline $\mathrm{CV} \%$ & \multicolumn{6}{|c|}{7.4} & \multicolumn{6}{|c|}{8.2} \\
\hline
\end{tabular}

Table 6. Combined analysis and interaction effects of urea doses and split applications on okra dry yield tons ha ${ }^{-1}$ over two seasons under rain-fed condition at Damazin Research Station.

\begin{tabular}{|c|c|c|c|c|c|c|c|c|c|c|c|c|}
\hline \multirow{3}{*}{ N levels (kg ha $\left.{ }^{-1}\right)$} & \multicolumn{6}{|c|}{ Season two } & \multicolumn{6}{|c|}{ Season one } \\
\hline & \multicolumn{6}{|c|}{ Splits doses } & \multirow[b]{2}{*}{0} & \multirow[b]{2}{*}{1} & \multirow[b]{2}{*}{2} & \multirow[b]{2}{*}{3} & \multirow[b]{2}{*}{4} & \multirow[b]{2}{*}{ Mean } \\
\hline & 0 & 1 & 2 & 3 & 4 & Mean & & & & & & \\
\hline 0 & 1.2 & & & & & 1.2 & 0.7 & & & & & 0.7 \\
\hline 43 & & 1.4 & 1.3 & 1.6 & 1.4 & 1.4 & & 1.2 & 1.1 & 1.4 & 1.2 & 1.2 \\
\hline 86 & & 1.7 & 1.8 & 1.8 & 1.2 & 1.6 & & 1.4 & 1.6 & 1.9 & 1.8 & 1.7 \\
\hline 129 & & 1.5 & 1.7 & 1.8 & 1.7 & 1.7 & & 1.9 & 2.0 & 1.9 & 1.4 & 1.8 \\
\hline Mean & 1.2 & 1.5 & 1.6 & 1.7 & 1.4 & & 0.7 & 1.5 & 1.6 & 1.7 & 1.5 & \\
\hline Statistics & \multicolumn{2}{|c|}{ Urea doses } & \multicolumn{2}{|c|}{ Splits doses } & \multicolumn{2}{|c|}{ Interaction } & \multicolumn{2}{|c|}{ Urea doses } & \multicolumn{2}{|c|}{ Splits doses } & \multicolumn{2}{|c|}{ Interaction } \\
\hline Sig. & \multicolumn{2}{|c|}{ * } & \multicolumn{2}{|c|}{$\star * *$} & \multicolumn{2}{|c|}{$* * *$} & \multicolumn{2}{|c|}{$* * *$} & \multicolumn{2}{|c|}{ * } & \multicolumn{2}{|c|}{$\star * *$} \\
\hline SE \pm & \multirow{2}{*}{\multicolumn{2}{|c|}{0.07}} & \multicolumn{2}{|c|}{0.05} & \multirow{2}{*}{\multicolumn{2}{|c|}{0.06}} & \multirow{2}{*}{\multicolumn{2}{|c|}{0.05}} & \multirow{2}{*}{\multicolumn{2}{|c|}{$\begin{array}{r}0.06 \\
8.2\end{array}$}} & \multirow{2}{*}{\multicolumn{2}{|c|}{0.1}} \\
\hline CV\% & & & \multicolumn{2}{|c|}{7.2} & & & & & & & & \\
\hline
\end{tabular}

\section{Conclusion}

According to these result presented it is suggested that the application of $86 \mathrm{~kg} \mathrm{ha}^{-1} \mathrm{~N}$ applied in two or three equal states starting at planting would yield appropriate okra fruit production under rain fed conditions in Blue Nile state, Sudan.

\section{REFERENCES}

Akinfasoye JA, Nwanguma El, 2005. Vegetative growth of Telfairia occidentialis Hook, F. and Staking pattern in telfaria/okra intercrop in a valley bottom dry season cultivation, In: Proceedings of the Horticultural Society of Nigeria Annual Conference held at the Rivers State College of Education, Port Harcourt, Nigeria. pp 67-71.

Buysse W, Stern R, Coe R, 2004. Gen Stat Discovery Edition for Everyday Use. ICRAF Nirobi, Kenya. 114 pp.

Cooper PJM, Leakey RRB, Rao MR, Reynolds L, 1996. Agroforestry and the mitigation of land degradation in the humid and sub-humid tropics of Africa. Exp Agric, 32:235-290.

FAOSTAT, 2010. Food and Agriculture Organization (FAO), Statistical
Data. 2010, FAO.http://faostat.fao.org/site/339/default.aspx accessed on $20 / 2 / 2013$.

Kolawole GO, Olapede AO, Alade CR, Olaniyi JO, 2008. Response of okra (Abelmoschus esculentus) varieties to NPK fertilizer in the South Guinea Savanna of Nigeria. Niger J Horticult Sci, 13: 99-108.

Omotoso SO, Shittu OS, 2007. Effect of NPK fertilizer rates and method of application on growth and yield of okra (Abelmoschus esculentus (L.) Moench). Res J Agron, 1(2): 84-87.

Osman EA, 2005. Field trials for the control okra leaf curl virus disease. Khartoum, Sudan (former): University of Khartoum, MSc thesis.

Rao MH, Subramanian TR, 1994. Fertilizer needs of vegetable crops based on yield goal approach in Alfisols in Southern India. J India Soc Soil Sci, 42(4): 565-568.

Tripathi KK, Govila OP, Warrier R, Ahuja V, 2011. Biology of Abelmoschus esculentus L. (Okra). Series of Crop Specific Biology Documents (35 p.), Department of Biotechnology, Ministry of Science \& Technology, \& Ministry of Environment and Forest, Govt. of India.

Citation: Adlan AMA, Adlan MA, Eisa YA, Amin El Sir Al, 2017. Effect of nitrogen rate and application on growth and yield of okra (Abelmoschus esculentus L.) under rain-fed conditions at Blue Nile State, Sudan. Net J Agric Sci, 5(4): 151-154. 